\title{
Figures
}

\section{Progression of neuroanatomical abnormalities after first-episode of psychosis: A 3-year longitudinal sMRI study}

\author{
Authors Theophilus N. Akudjedu ${ }^{a, b^{*}}$, Giulia Tronchin ${ }^{a}$, Shane Mclnerney ${ }^{a, f}$, Cathy Scanlon ${ }^{a}$, Joanne P.M. \\ Kenney e, John McFarland ${ }^{a}$, Gareth J. Barker ${ }^{d}$, Peter McCarthy ${ }^{c}$, Dara M. Cannon ${ }^{a}$, Colm McDonald $^{a}$, \\ Brian Hallahan ${ }^{a}$
}

\begin{abstract}
Affiliations ${ }^{a}$ Centre for Neuroimaging \& Cognitive Genomics (NICOG), Clinical Neuroimaging Laboratory, NCBES Galway Neuroscience Centre, College of Medicine Nursing and Health Sciences, National University of Ireland Galway, H91TK33 Galway, Ireland. 'Institute of Medical Imaging \& Visualisation, Department of Medical Science and Public Health, Faculty of Health and Social Sciences, Bournemouth University, Bournemouth, UK. 'Department of Radiology, College of Medicine Nursing and Health Sciences, National University of Ireland Galway, H91TK33 Galway, Ireland. 'King's College London, Institute of Psychiatry, Psychology \& Neuroscience, Department of Neuroimaging, London, UK. 'Trinity College Institute of Neuroscience and School of Psychology, Trinity College Dublin, Dublin, Ireland. 'Department of Psychiatry, University of Toronto, 250 College Street, 8th floor, Toronto, Canada.
\end{abstract}

\section{Corresponding Author*}

Theophilus N. Akudjedu

Institute of Medical Imaging \& Visualisation,

Department of Medical Science and Public Health, Faculty of Health and Social Sciences,

Bournemouth University,

Bournemouth, UK.

Email takudjedu@bournemouth.ac.uk 
Fig.1: Progressive volume change over time in FEP patients and controls
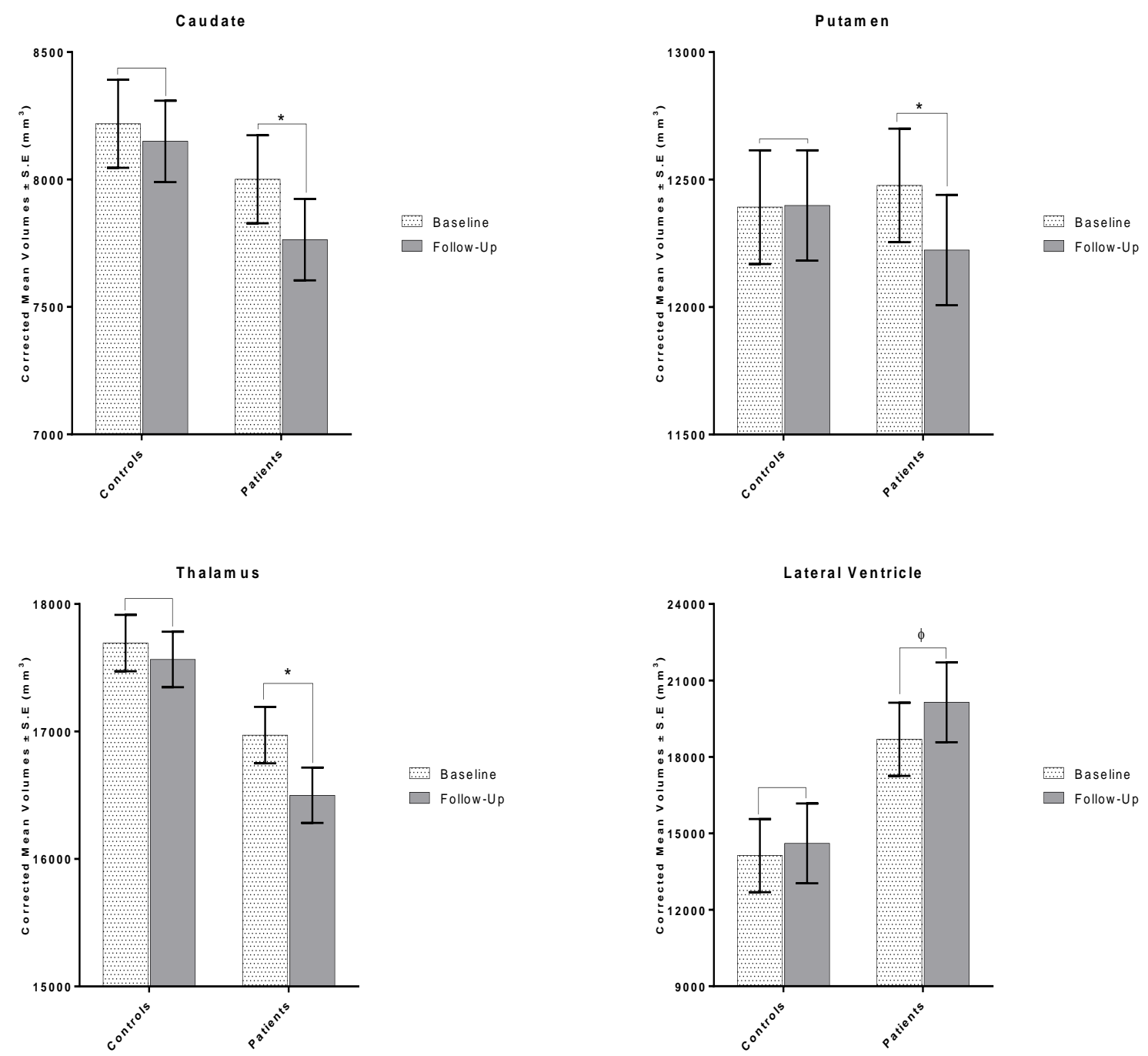

Fig.1 Legend: Plot of corrected mean volumes ( \pm standard error) of the neuroanatomical structures that progressed overtime. Comparison of these progressions in first-episode psychosis patients with healthy controls at baseline and after 3-years. The mean volumes were corrected for ICV, gender and age at baseline. *significant change; $\$$ greater progressive change that did not reach statistical significance. 
Fig. 2: Corrected $p$-value maps showing regional neuroanatomical clusters withincreased symmetrised rates of progressive cortical thinning in FEP patients relative to HCs over time.Cluster-wise correction for multiple comparison at $\mathrm{p}=0.05$.

Left Hemisphere

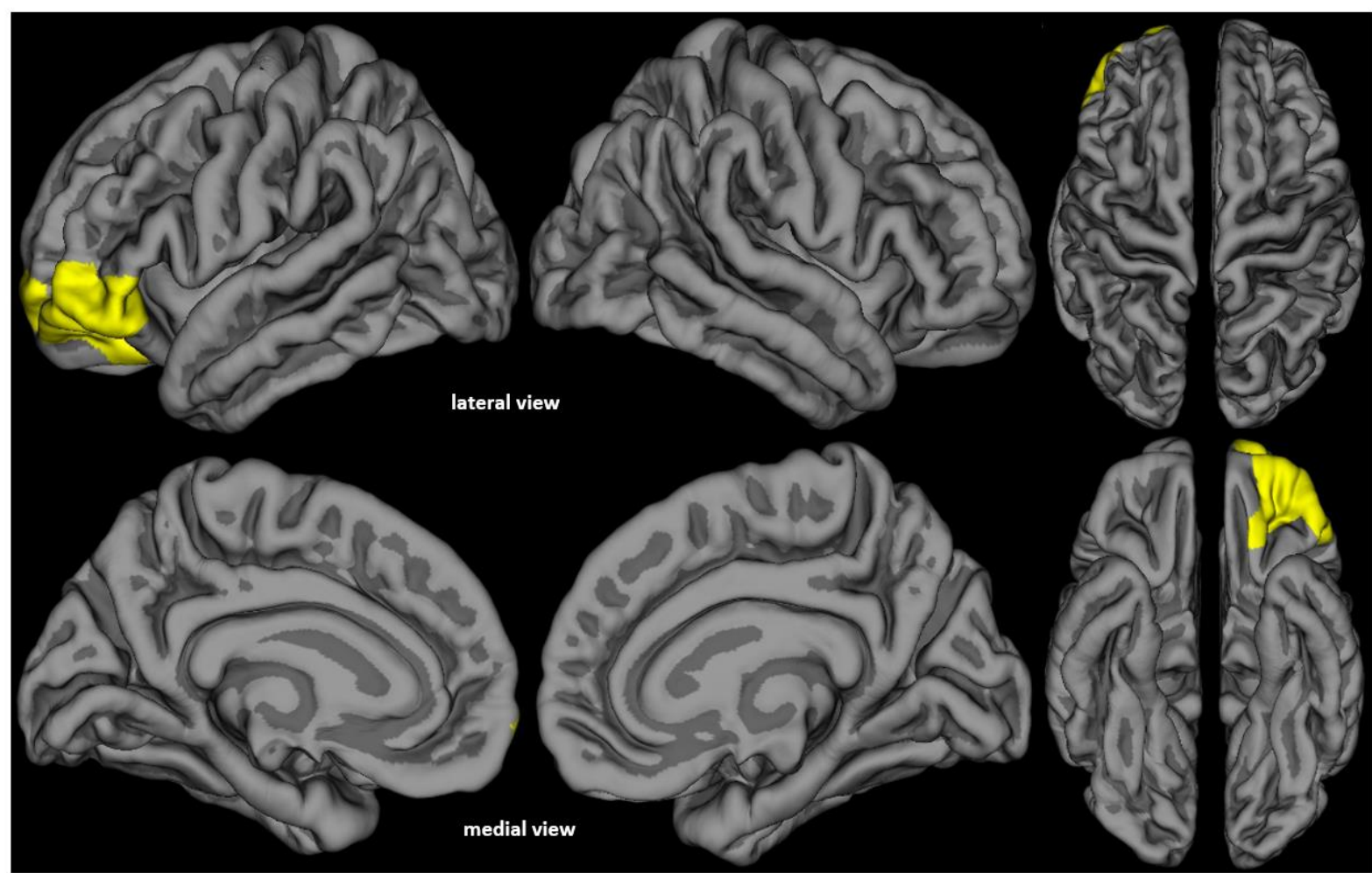

Right Hemisphere

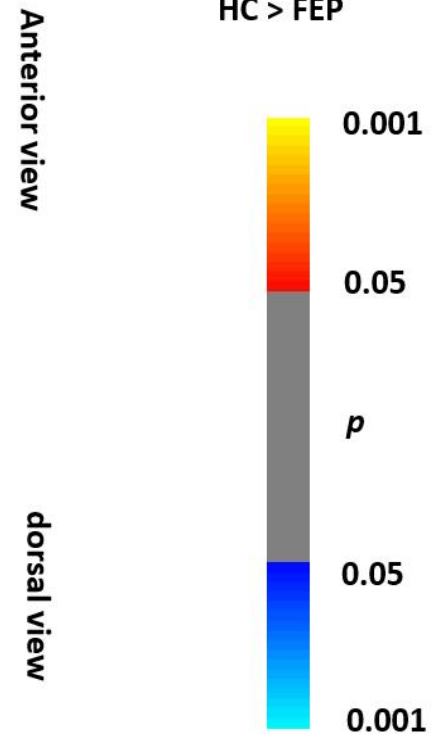

Fig. 2 Legend: The symmetrised rate of progressive cortical thickness change in FEP patients relative to healthy controls per year. The regional neuroanatomical clusters that survived cluster-wise correction for multiple comparison $(p=0.05)$ for cortical thinning coincided with the LLOFR with a cluster probability $p<0.0001$ with Talairach coordinates of maxima $(-25.3,42.1,-10.1)$ are displayed in YELLOW. This region coincides with the left lateral orbitofrontal cortex extending into aspects of the left pars orbitalis, pars triangularis, rostral middle frontal gyrus and frontal pole. Of note, when the 4 patients on mood stabilisers at follow-up were removed from the analyses, these findings remained essentially unaltered. 